CSD refcodes (Refcode) and bond valence parmaters (R) for La(III)-C bonds.

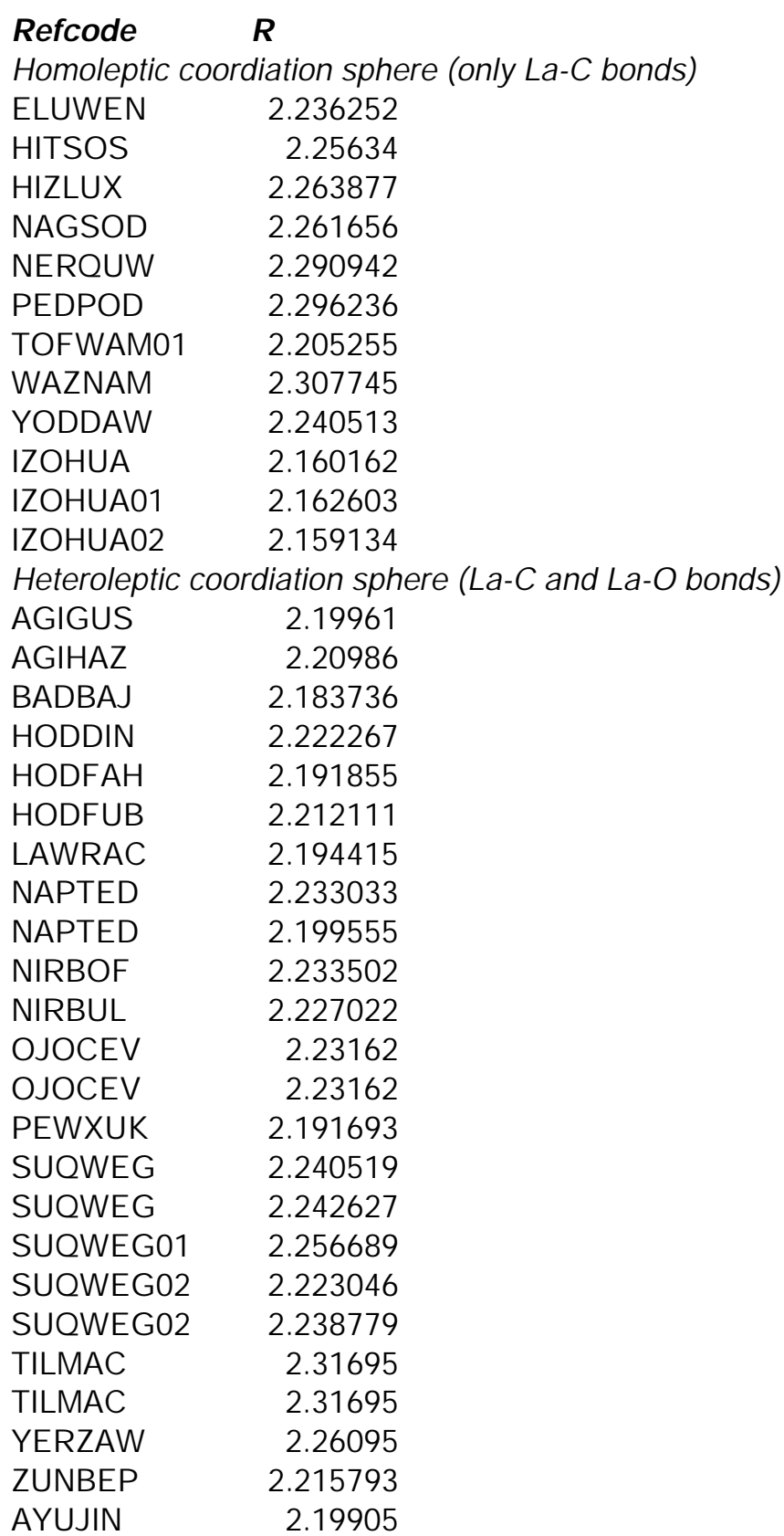


CSD refcodes (Refcode) and bond valence parmaters (R ) for Ce(III)-C bonds.

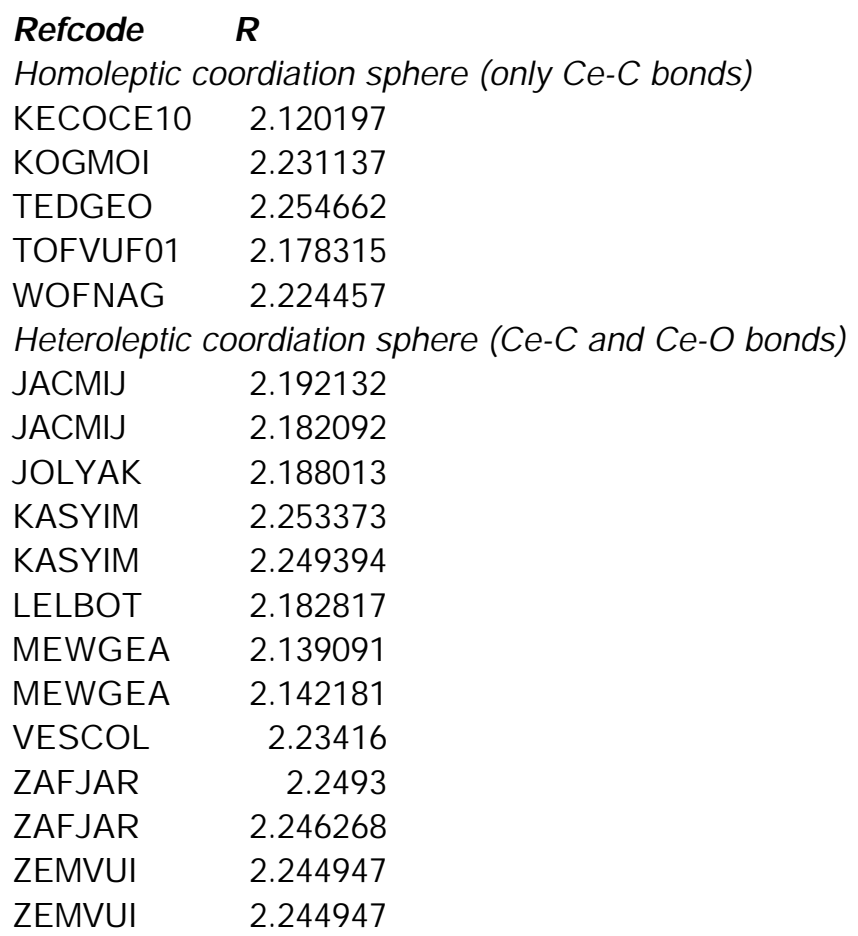


CSD refcodes (Refcode) and bond valence parmaters (R ) for $\operatorname{Pr}(I I I)-C$ bonds.

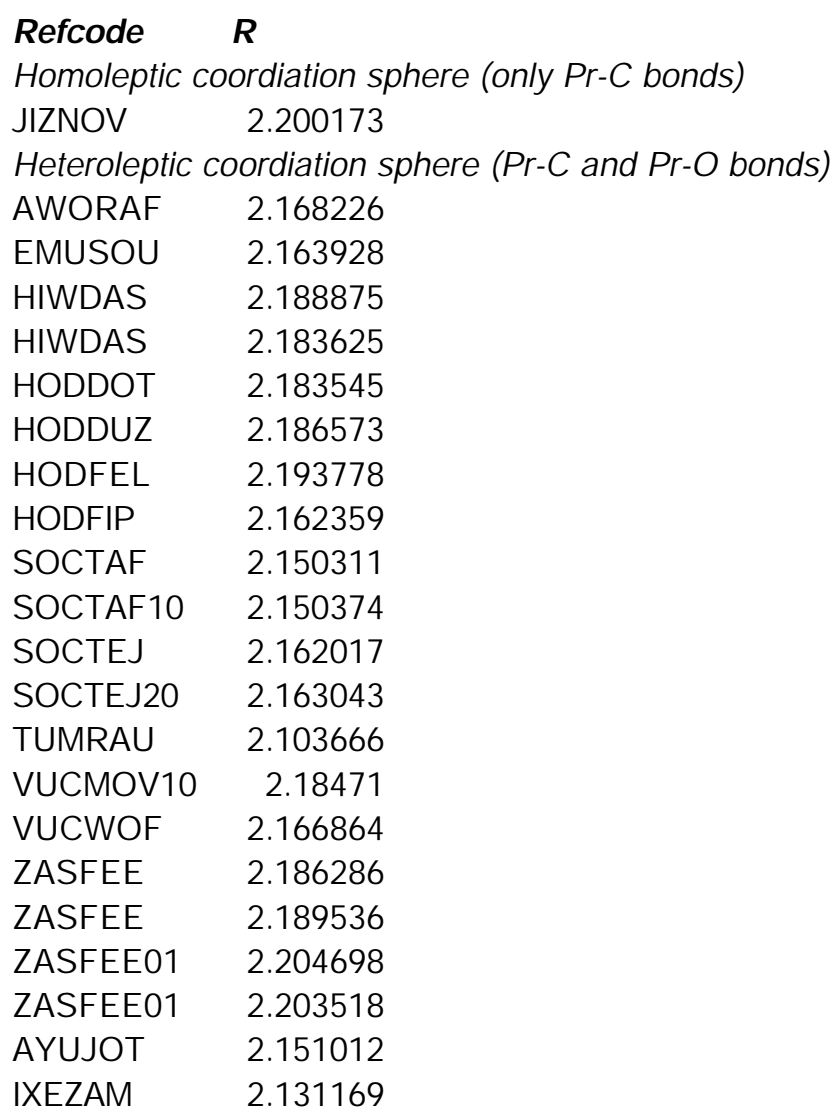


CSD refcodes (Refcode) and bond valence parmaters ( $R$ ) for $\mathrm{Nd}(\mathrm{III})-\mathrm{C}$ bonds.

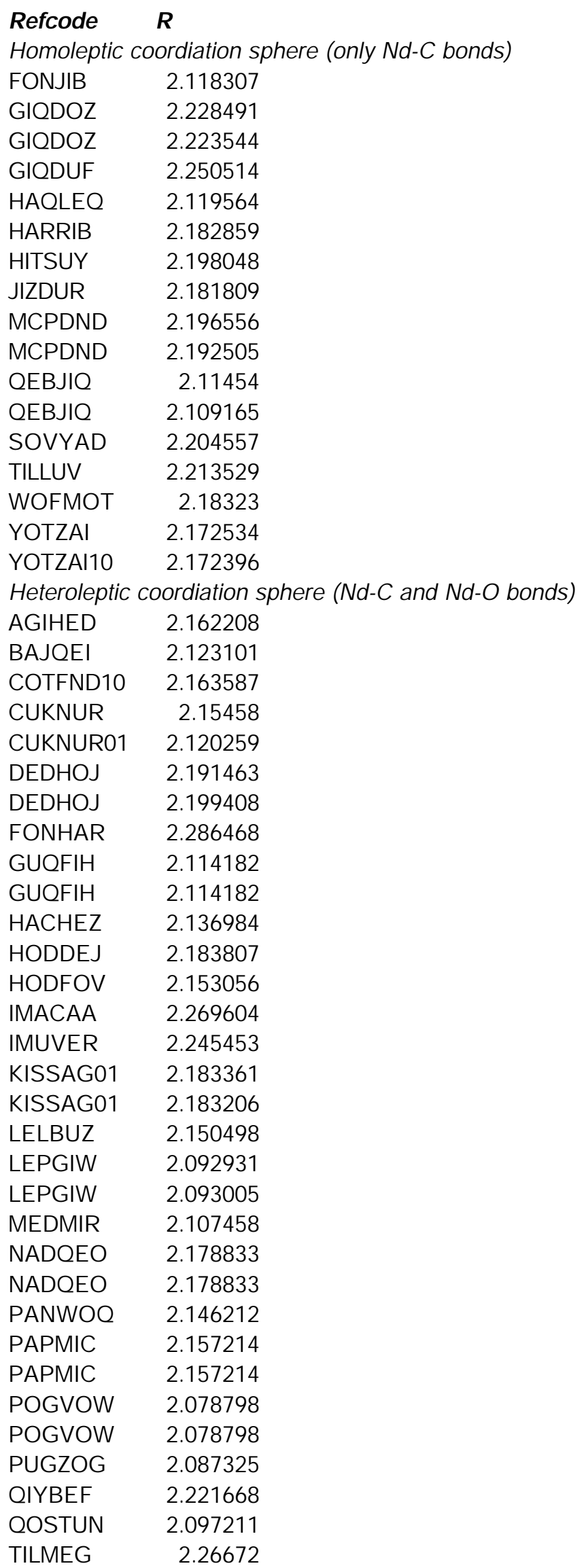




$\begin{array}{lr}\text { TILMEG } & 2.26672 \\ \text { TILMEG01 } & 2.295534 \\ \text { TIMQOV } & 2.135105 \\ \text { VIVBOR } & 2.154186 \\ \text { WAGWUW } & 2.158391 \\ \text { WEFPUS } & 2.133475 \\ \text { WEYRAT } & 2.142722 \\ \text { YEZXUW } & 2.125215 \\ \text { YIYFOB } & 2.099828 \\ \text { YOPMEV } & 2.302125 \\ \text { YUWDID } & 2.121789 \\ \text { ZEMWAP } & 2.305127 \\ \text { ZUNBOZ } & 2.163291 \\ \text { PAGNAN } & 1.894682 \\ \text { PAGNAN } & 1.894682 \\ \text { PAGNER } & 2.137674 \\ \text { PAGNIV } & 2.139946 \\ \text { RAFCUX } & 2.205573\end{array}$


CSD refcodes (Refcode) and bond valence parmaters ( $R$ ) for $\mathrm{Nd}(\mathrm{III})-\mathrm{C}$ bonds.

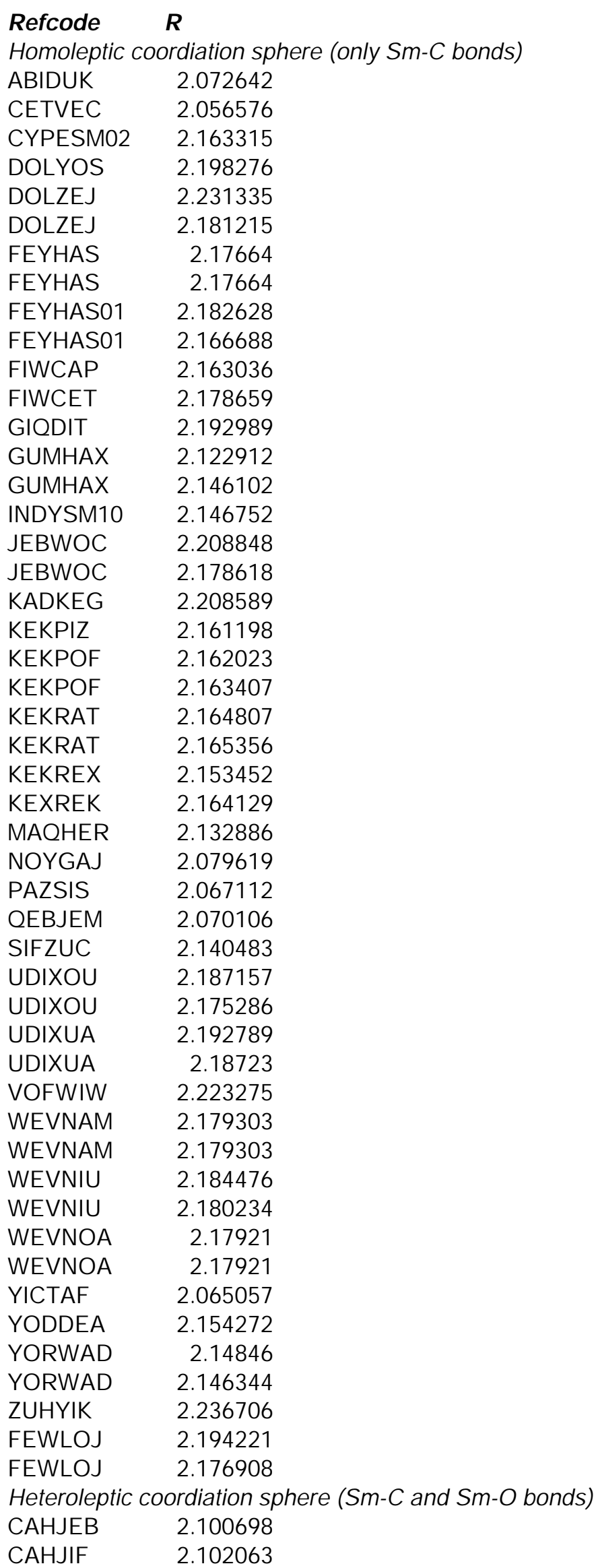




\begin{tabular}{lr} 
CUPSEL & 2.133597 \\
CUPSEL & 2.149232 \\
CUPSIP & 2.105892 \\
CUPSIP & 2.102904 \\
CUWGEG & 2.163992 \\
CUWGEG & 2.163992 \\
DIGJEI & 2.118113 \\
DOLYUY & 2.235402 \\
DOMGER & 2.117016 \\
DOMGER & 2.117016 \\
GACSEI & 2.126568 \\
GACSEI & 2.120582 \\
GISLUP & 2.034693 \\
GISMAW & 2.060348 \\
HEQJES & 2.10866 \\
IHECED & 2.151611 \\
JACLUV & 2.158968 \\
JACLUV & 2.158968 \\
JAQDOU & 2.155458 \\
JAQDUA & 2.150134 \\
JEYNEG & 2.109923 \\
JEYNEG & 2.109923 \\
KEKPUL & 2.18614 \\
KEWNOP & 2.197153 \\
KEWPAD & 2.109572 \\
KILMUN & 2.135682 \\
KILMUN & 2.135682 \\
KONYUH & 2.161956 \\
KONYUH & 2.161956 \\
KONYUHO1 & 2.149991 \\
KONYUHO1 & 2.149991 \\
KONYUHO2 & 2.15103 \\
KONYUHO2 & 2.15103 \\
LAYRUY & 2.148818 \\
LIQKUR & 2.1943 \\
LIQKUR & 2.160137 \\
MAVBAM & 2.199131 \\
MAVBEQ & 2.111904 \\
NAPTIH & 2.126145 \\
NAPTIH & 2.126145 \\
OJOCOF & 2.177216 \\
POHOCOF & 2.172588 \\
POHFIB & 2.133663 \\
PUUUG & 2.153646 \\
PUNXUX & 2.104792 \\
PEUG & 2.153646 \\
RABTOZ & 2.11503 \\
PIGYOT & 2.113048 \\
PIGYOTO1 & 2.108118 \\
PIGYOT10 & 2.111486 \\
POGVUC & 2.062024 \\
POGVUC & 2.060142 \\
POGVUC & 2.062024 \\
\hline
\end{tabular}




$\begin{array}{lr}\text { RABTOZ } & 2.11503 \\ \text { SESGUS } & 2.131639 \\ \text { SESGUS } & 2.131639 \\ \text { SESKAC } & 2.118115 \\ \text { SESKAC } & 2.118115 \\ \text { SESKEG } & 2.118097 \\ \text { SESKEG } & 2.118097 \\ \text { TAMWUZ } & 2.128921 \\ \text { TAMWUZO1 } & 2.131031 \\ \text { TATDIB } & 2.143976 \\ \text { TATDIB } & 2.142035 \\ \text { TINGOM } & 2.097269 \\ \text { VIQTIY } & 2.14669 \\ \text { VIQTOE } & 2.150523 \\ \text { VIQTOE } & 2.136801 \\ \text { VIZTON } & 2.109686 \\ \text { VIZTON } & 2.109686 \\ \text { VULFAJ } & 2.120371 \\ \text { ZIWROM } & 2.140134 \\ \text { DANQAL } & 2.120637 \\ \text { DANQAL } & 2.140888\end{array}$


CSD refcodes (Refcode) and bond valence parmaters (R ) for Eu(III)-C bonds.

\section{Refcode $\mathbf{R}$}

Heteroleptic coordiation sphere (Eu-C and Eu-O bonds)

BIDREL 2.065451

BIDREL 2.065451

YEBXAE 2.224993

YEBXAE $\quad 2.184005$ 
CSD refcodes (Refcode) and bond valence parmaters (R ) for $\mathrm{Gd}(\mathrm{III})$-C bonds.

\section{Refcode $\quad \mathbf{R}$}

Homoleptic coordiation sphere (only $\mathrm{Gd}-\mathrm{C}$ bonds)

FOGJEQ 2.116883

HITTAF 2.142231

KIFTUO 2.176869

Heteroleptic coordiation sphere ( $\mathrm{G} d-\mathrm{C}$ and $\mathrm{Gd}-\mathrm{O}$ bonds)

CPTHGD10 2.104336

OJ OCIZ 2.164373

OJ OCIZ 2.155778

YUWDEZ 2.096233

YUWDEZ 2.047338

ZUNBUF $\quad 2.121984$

EWURUJ 2.058227 
CSD refcodes (Refcode) and bond valence parmaters (R ) for Tb(III)-C bonds.

Refcode $\mathbf{R}$

Homoleptic coordiation sphere (only Tb-C bonds)

LAFVIX 2.120324

PEGYAB 2.034812 
CSD refcodes (Refcode) and bond valence parmaters (R ) for Dy(III)-C bonds.

\section{Refcode $\mathbf{R}$}

Homoleptic coordiation sphere (only Dy-C bonds)

ABIFAS 2.026342

HITTEJ 2.114499

MOXBIK 2.159623

Heteroleptic coordiation sphere (Dy-C and Dy-O bonds)

KILZOU 2.118611

KILZOU02 2.06996

PAPMOI 2.076761

PAPMOI 2.076761

TEGKAR 2.08946

YEGNAZ 2.015784

YEGNAZ 2.015784

YEZYIL $\quad 2.068601$

YEZYIL $\quad 2.068601$

BIFNEK 2.04943 
CSD refcodes (Refcode) and bond valence parmaters (R ) for Ho(III)-C bonds.

Refcode R

Heteroleptic coordiation sphere ( $\mathrm{Ho}-\mathrm{C}$ and $\mathrm{Ho}-\mathrm{O}$ bonds)

ULOVUM 2.072927

WEXWEB 2.049047 
CSD refcodes (Refcode) and bond valence parmaters (R) for Er(III)-C bonds.

\section{Refcode $\mathbf{R}$}

Homoleptic coordiation sphere (only Er-C bonds)

ABIFEW 2.000614

FABKUO 2.07117

FABKUO 2.093944

Heteroleptic coordiation sphere ( $\mathrm{Er}-\mathrm{C}$ and $\mathrm{Er}-\mathrm{O}$ bonds)

J AQFAI 2.070819

JUSDEG 2.055584

JUSDEG $\quad 2.055584$

PANYAE $\quad 2.070489$

YEDSIJ $10 \quad 2.078534$

YEZYAD 2.028193 
CSD refcodes (Refcode) and bond valence parmaters (R ) for Tm(III)-C bonds.

\section{Refcode R}

Homoleptic coordiation sphere (only $\mathrm{Tm}-\mathrm{C}$ bonds)

QQQCASC 2.06605

QQQCASC 2.073899

RACYUQ $\quad 1.99191$

WOFMUZ 2.076306

Heteroleptic coordiation sphere ( $\mathrm{Tm}-\mathrm{C}$ and $\mathrm{Tm}-\mathrm{O}$ bonds)

RIRQOY 2.03627

RIRQOY 2.03627 
CSD refcodes (Refcode) and bond valence parmaters ( $R$ ) for $Y b(I I I)-C$ bonds.

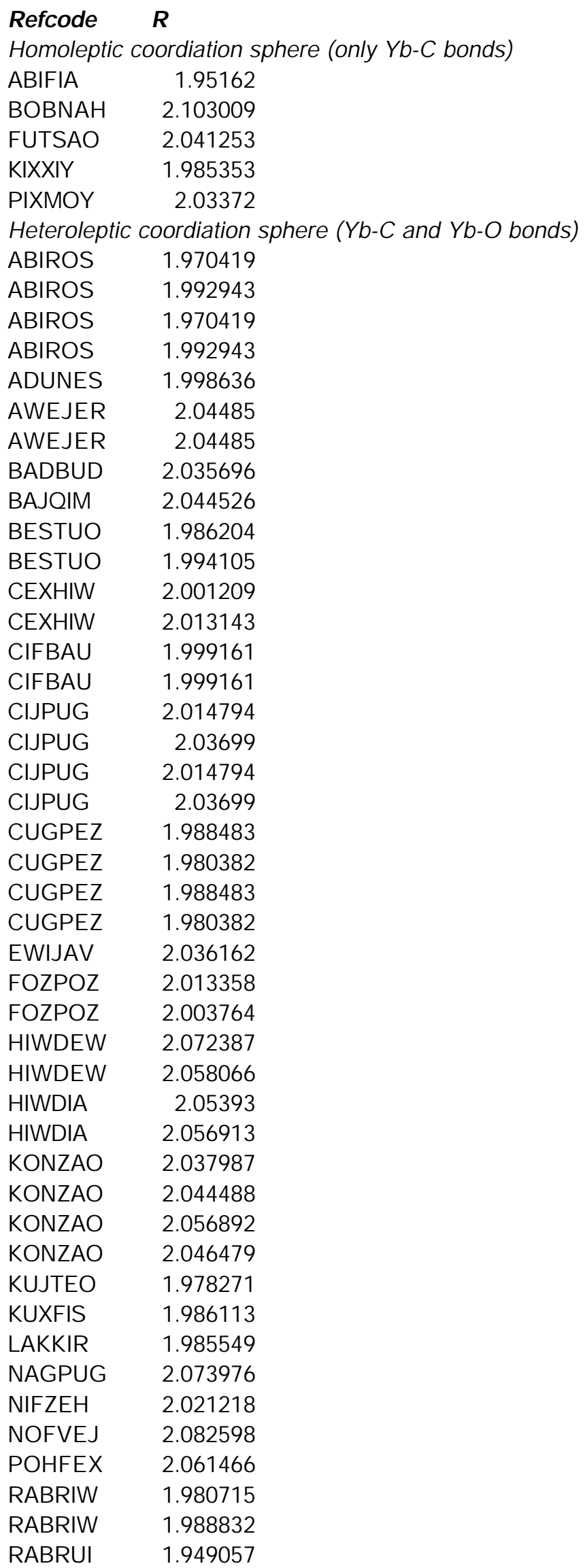




$\begin{array}{lr}\text { RABRUI } & 1.963965 \\ \text { RABRUI01 } & 1.952624 \\ \text { RABRUI01 } & 1.97864 \\ \text { TAKSUU } & 2.093048 \\ \text { VIMBIC } & 2.031186 \\ \text { VIMBIC } & 2.031186 \\ \text { VUBPAJ } & 1.962064 \\ \text { VUBPAJ } & 1.962064 \\ \text { WACTEZ } & 1.98714 \\ \text { WACTEZ } & 1.98714 \\ \text { WACTEZ10 } & 1.999193 \\ \text { WACTEZ10 } & 1.999193 \\ \text { WACTEZ20 } & 1.98714 \\ \text { WACTEZ20 } & 1.98714 \\ \text { WIBHEU } & 1.977482 \\ \text { YAXLEO } & 2.045454 \\ \text { YAXLEO } & 2.029982 \\ \text { YEZYOR } & 2.032719 \\ \text { YEZYOR } & 2.032719 \\ \text { ZIVYEI } & 1.978806 \\ \text { ZIVYEI } & 1.978806 \\ \text { ZIVYIM } & 1.999287 \\ \text { ZIVYIM } & 1.999287 \\ \text { ZOFCAY } & 1.991864 \\ \text { ZOFCAY } & 1.968205 \\ \text { ZOFCAY } & 1.991864 \\ \text { ZOFCAY } & 1.968205 \\ \text { ZOTZEN } & 1.92473 \\ \text { ZUBDOP } & 1.996305\end{array}$


CSD refcodes (Refcode) and bond valence parmaters (R) for Lu(III)-C bonds.

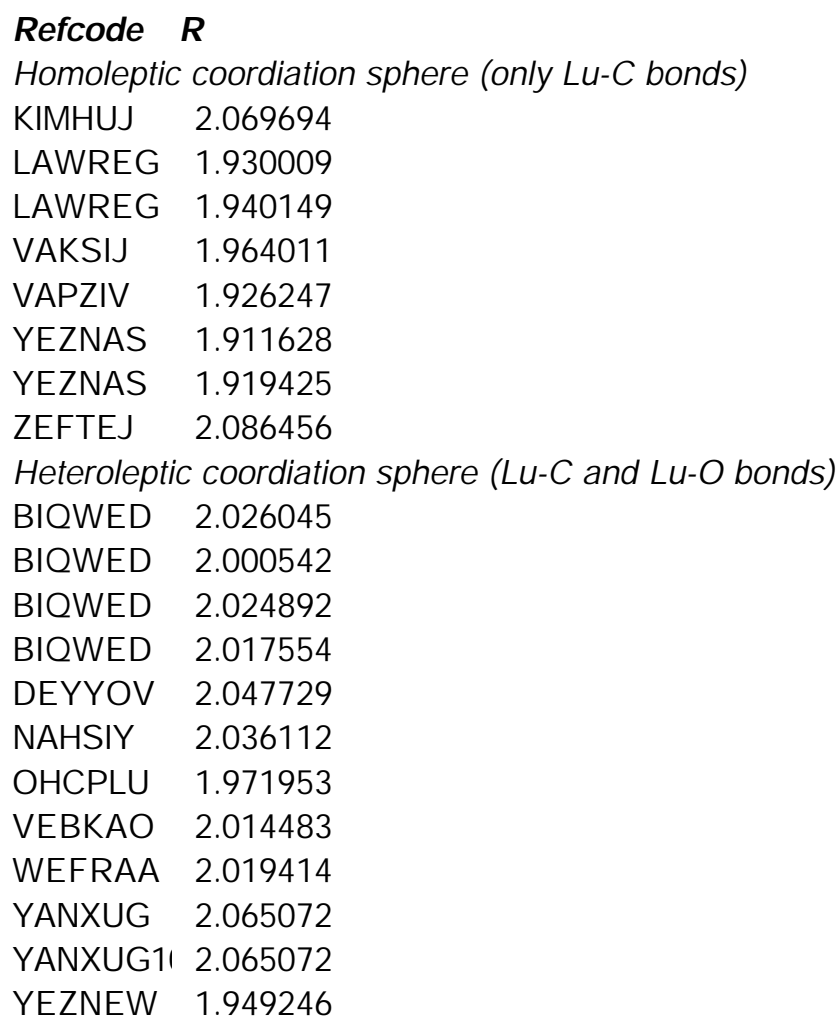

